\title{
A robust strategy for optimizing complex distillation columns
}

\author{
Filipe J.M. Neves, Dulce C.M. Silva, Nuno M.C. Oliveira* \\ GEPSI-PSE Group, Department of Chemical Engineering, University of Coimbra, Pólo II, Pinhal de Marrocos, 3030-290 Coimbra, Portugal
}

Available online 16 March 2005

\begin{abstract}
This work introduces a strategy for the optimal design of distillation systems based on continuous optimization. The approach is similar to the one proposed earlier by [Lang, Y. -D., \& Biegler, L. T. (2002). A distributed stream method for tray optimization. AIChE Journal, 48, 582], avoiding the need of solving extremely large and non-linear discrete optimization problems. When used with complex distillation units, it can identify interesting design configurations not considered by other continuous formulations, and also relieve some of the numerical difficulties associated with the use of distribution functions for the optimal location of feed and side-streams. The method considers a relaxation of the original problem, where the streams are initially split to several trays in the column, not necessarily adjacent. The optimal location of each stream is converged by constraining the optimization problem, using adjustable parameters that control the minimum amount of aggregation allowed. The methodology is illustrated with the application to several industrial case studies, including sets of distillation columns. Models up to 17,000 variables/equations were solved, revealing large economic benefits in the design of new units and optimization of sets of existing ones.
\end{abstract}

(C) 2005 Published by Elsevier Ltd.

Keywords: Distillation; Optimization; Continuous formulations; Relaxation

\section{Introduction}

The topic of optimization of distillation columns has received significant attention in the past decades due, at the same time, to its economical importance and the numerical difficulties associated with the solution of this type of problems. Among the difficulties usually encountered, it is possible to emphasize:

(a) The complexity of the models required to adequately describe the equilibrium phenomenon that takes place. The use of detailed non-ideal equilibrium models, such as the UNIFAC group contribution method, is often necessary (Reid, Prausnitz, \& Poling, 1987). When the nonideality of the vapor and liquid phases is simultaneously considered, the corresponding models can require up to 50 scalar variables per component per equilibrium stage, leading easily to overall unit models with tens of thou-

\footnotetext{
* Corresponding author. Tel.: +351239 798700; fax: +351 239798703.

E-mail addresses: fneves@eq.uc.pt (F.J.M. Neves), dulce@eq.uc.pt (D.C.M. Silva), nuno@eq.uc.pt (N.M.C. Oliveira).
}

sands of non-linear algebraic equations, and highly nonlinear behavior.

(b) The need to incorporate discrete decisions in the solution process, related to the optimal location of the feed and product streams, and the total number of equilibrium stages. These problems are usually addressed as mixed-integer non-linear programs-MINLP (Barttfeld \& Aguirre, 2002; Barttfeld, Aguirre, \& Grossmann, 2003; Bauer \& Stilchmair, 1998; Viswanathan \& Grossmann, 1990, 1993) or general disjunctive programs_GDP (Barttfeld et al., 2003; Yeomans \& Grossmann, 2000).

A common difficulty associated with the use of MINLP formulations is the need to satisfy each model constraint, even in cases where a particular equilibrium stage is eliminated from the correspondent superstructure. This can lead to models of large size, where singularities can be encountered during the integer solution phase, especially associated with linearizations at zero flows. These characteristics affect adversely the robustness of MINLP approaches and constituted the main motivation for the development of alternative 


\begin{tabular}{|c|c|}
\hline \multicolumn{2}{|c|}{ Nomenclature } \\
\hline$A$ & area $\left(\mathrm{m}^{2}\right)$ \\
\hline$b$ & $\begin{array}{l}\text { variable associated to the fractioning of a } \\
\text { stream }\end{array}$ \\
\hline$C$ & annualized cost $(€ /$ year $)$ \\
\hline$D$ & column diameter $(\mathrm{m})$ \\
\hline$F$ & feed flow rate $(\mathrm{mol} / \mathrm{h})$ \\
\hline$h$ & column height $(\mathrm{m})$ \\
\hline$H$ & enthalpy $(\mathrm{J} / \mathrm{mol})$ \\
\hline$K$ & liquid-vapor equilibrium "constant" \\
\hline$L$ & liquid flow rate $(\mathrm{mol} / \mathrm{h})$ \\
\hline$n$ & total number \\
\hline$Q$ & heat exchanged $(\mathrm{J} / \mathrm{h})$ \\
\hline$R$ & reflux ratio \\
\hline$S$ & operational specification in a side-stream \\
\hline$T$ & temperature $(\mathrm{K})$ \\
\hline$U$ & liquid side-stream flow rate $(\mathrm{mol} / \mathrm{h})$ \\
\hline$V$ & vapor flow rate $(\mathrm{mol} / \mathrm{h})$ \\
\hline$x$ & liquid phase composition (mole fraction) \\
\hline$y$ & vapor phase composition (mole fraction) \\
\hline$W$ & vapor side-stream flow rate $(\mathrm{mol} / \mathrm{h})$ \\
\hline \multicolumn{2}{|c|}{ Greek letters } \\
\hline$\alpha$ & $\begin{array}{l}\text { adjustable parameter used to force streams to } \\
\text { enter in one plate }\end{array}$ \\
\hline$\varepsilon$ & $\begin{array}{l}\text { slack variables concerning the MESH equa- } \\
\text { tions }\end{array}$ \\
\hline$\mu$ & $\begin{array}{l}\text { stream location central value in the DDF strat- } \\
\text { egy }\end{array}$ \\
\hline$\delta$ & $\begin{array}{l}\text { tolerance concerning the error admissible in the } \\
\text { MESH equations }\end{array}$ \\
\hline \multicolumn{2}{|c|}{ Subscripts } \\
\hline $\mathrm{BE}$ & relative to the energy balance equations \\
\hline BMP & relative to the partial mass balance equations \\
\hline BMT & relative to the total mass balance equations \\
\hline c & relative to components \\
\hline $\mathrm{C}$ & relative to the condenser \\
\hline $\mathrm{CU}$ & relative to cold utility \\
\hline EQ & relative to the equilibrium equations \\
\hline $\mathrm{F}$ & relative to the feed \\
\hline $\mathrm{HE}$ & relative to heat exchanging equipment \\
\hline HU & relative to hot utility \\
\hline$i$ & relative to component $i$ \\
\hline$j$ & relative do plate $j$ \\
\hline $\mathrm{L}$ & relative to a liquid phase \\
\hline LS & relative to liquid side-stream \\
\hline $\max$ & relative to a maximum allowed value \\
\hline & relative to a minimum allowed value \\
\hline opt & relative to an optimal obtained value \\
\hline $\mathrm{p}$ & relative to plates \\
\hline & relative to the reboiler \\
\hline Reb & relative to the reboiler \\
\hline
\end{tabular}

\begin{tabular}{|ll|}
\hline & \\
Ref & relative to the reflux \\
SI & relative to the shell and internals of a columns \\
T & relative to a total quantity \\
V & relative to a vapor phase \\
VS & relative to vapor side-stream \\
0 & relative to a constant/imposed flow-rate \\
\end{tabular}

GDP formulations. Contrarily to MINLP, GDP models use logic constraints to select a given subset of model equations to be satisfied, thus requiring the solution of smaller NLP subproblems, which can be converged more reliably. An important property of MINLP formulations for distillation is that they often possess continuous relaxations that constitute good approximations of the true integer solution (Grossmann, Aguirre, \& Barttfeld, 2004). This property, observed on some of the examples considered here, is not exploited by the GDP approach, since in this case only NLP subproblems with a fixed number of equilibrium stages are solved. Perhaps the most important limitation on the use of these discrete formulations, from a practical point of view, is the still limited choice of numerical solvers available for these types of problems, associated with moderate numerical robustness and computational requirements that can be extremely dependent on proper initialization and bounding of the problem.

Recently, Lang and Biegler (2002) introduced a strategy for column optimization that avoids the use of discrete formulations. This strategy has the advantage of requiring only the solution of continuous optimization problems (NLP), allowing therefore the use of more robust numerical solvers available for this kind of problems. The main idea of this approach is to employ a differentiable distribution function (DDF), characterized by a central value and a dispersion factor to locate the most appropriate region for a given stream in the column. The method uses the solution of a sequence of closely related optimization problems with increasingly narrower dispersion factors to find the optimal locations for feed and product streams, as well as the optimal total number of equilibrium stages. Similarly, to the relation between MINLP and GDP methods, the DDF approach can be refined by the formulation of the corresponding continuous design problem as a mathematical program with equilibrium constraints or MPEC (Raghunathan \& Biegler, 2003). This modification allows the bypass of the model equations for the trays that become inactive, by introduction of appropriate complementarity conditions. However, this approach requires the use of specific software for the solution of MPECs, which is currently an area of very active development (Raghunathan \& Biegler, 2004).

An alternative continuous strategy for the optimal design of a column or a fixed configuration of a set of distillation columns is presented in Section 2 of the paper. Instead of relying on distribution functions, it introduces simple constraints 
used to control the amount of stream splitting, and to converge the relaxation of the initial design problem to its optimal solution. Section 3 discusses critical aspects for the successful application of continuous optimization to large-scale and complex distillation problems, namely the pre-processing phase and further modeling aspects. Section 4 presents the complete mathematical formulation considered. In Section 5, illustrative examples are used to discuss the main details associated with this new strategy. Several large-scale examples of industrial applications are chosen to test the numerical robustness of the overall procedure. We show that the present approach can suggest interesting column configurations not considered by its predecessor. The paper ends with conclusions and identification of areas for future development.

\section{Continuous formulations for optimization of distillation columns}

A strategy for the optimization of distillation columns based on the concept of differentiable distribution functions was recently proposed (Lang \& Biegler, 2002). This is illustrated in Fig. 1(a). In this method, each stream $i$ to be optimally located is associated with a $\mathrm{DDF}_{i}$, characterized by a central value $\left(\mu_{i}\right)$ and a dispersion factor $\left(\sigma_{i}\right)$. The parameter $\sigma$ can be regarded as expressing the uncertainty associated with the location of a particular stream, at a given iteration step. Thus, for a set of fixed parameters $\sigma$ (one per stream), a continuous optimization problem is solved, producing the optimal estimates of the central values relative to each stream.

The method requires the solution of a sequence of optimization problems, with increasingly narrower distributions, to converge the stream locations to single equilibrium stages. It should be noted that, in this procedure, it is also possible to fix each $\sigma_{i}$ at a value that will guarantee that only one tray will be selected, and therefore formulate only one optimization problem for the optimal column design. However, the available computational experience shows that, in general, this leads to an extremely non-linear problem, very susceptible to the presence of local optima, requiring the use of global NLP algorithms. For this reason, sufficiently large values of the parameters $\sigma$ are instead considered during the early solution phases, producing a wide distribution that covers significantly well all of the candidate trays of interest. As the solution proceeds, smaller values of $\sigma$ are progressively introduced, leading to narrower distributions, and therefore to the iterative optimal location of the stream, eventually converging to a single equilibrium stage. This can be done much more easily using gradient-based NLP solvers, as the examples presented here and by Lang and Biegler (2002) show.

An interesting property of this approach is the relation of its intermediate solutions with the continuous relaxations of the corresponding MINLP formulations, described earlier. As mentioned before, the NLP relaxations of the MINLP problems constitute often good approximations of the true integer solutions. For simple columns, where the continuous relaxations tend to distribute the streams by a few adjacent trays, the initial solutions produced by the DDF approach tend also to be close to the optimum, and therefore require only few iterations, provided that the "shape" of the distribution function and the dispersion factor $\sigma$ are carefully tuned. However, for more complex columns or column arrangements, that might benefit from stream splitting and their introduction in different points of the columns, the solutions produced by the DDF approach can be quite different from the correspondent

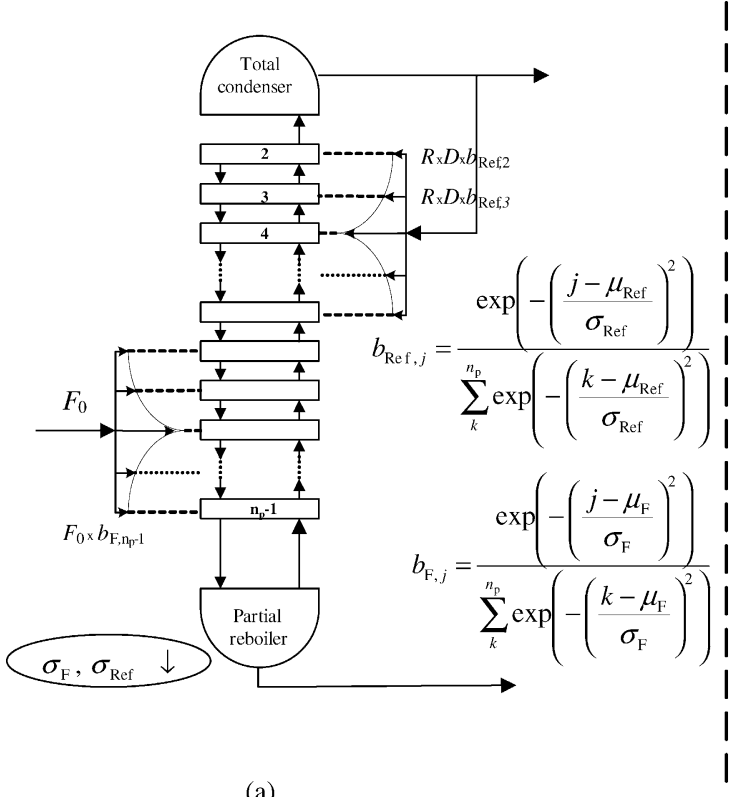

(a)

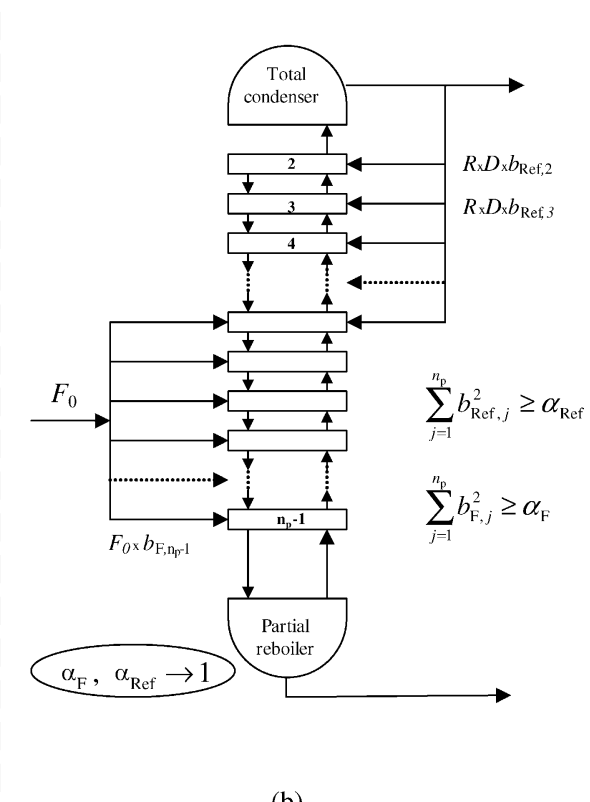

(b)

Fig. 1. Continuous formulations for the optimization of distillation units (a) DDF-based and (b) split fractions-based. 
MINLP relaxations, as illustrated by some of the examples considered in this paper. In this case, the efficient use of the DDF approach requires a priori knowledge of whether and where this type of more complex arrangements might be advantageous, to produce comparable solutions to the MINLP approach.

A further aspect of the DDF approach is that, due to their nature, the equations introduced by this method can present high sensitivities to changes in the continuous variable representing the location of the feed or product streams $\left(\mathrm{DDF}_{i}\right)$, for the range of distribution parameters $\sigma$ necessary to discriminate individual optimal locations. This might potentially lead to ill-conditioning, or high numerical sensitivity in the resulting optimization models, and therefore to numerical difficulties in more demanding cases.

An alternative formulation, also based on the use of only continuous variables is presented in Fig. 1(b). Here, we try to combine the most important advantages of both previous discrete and continuous approaches. This is done by solving only NLP subproblems and retaining a greater similarity between the intermediate solutions and the continuous relaxations of the corresponding discrete formulations. In this approach, similar to the DDF case, the feed and product streams are initially distributed to each tray of the column, using continuous variables $\left(b_{\mathrm{F}, j}\right.$ and $b_{\mathrm{Ref}, j}$ in the case of Fig. 1(b)). These variables represent the split fraction of a given stream $i$ (feed or product) among the different equilibrium stages of the column, and therefore are subject to conservation constraints of the form

$$
\begin{gathered}
\sum_{j=1}^{n_{\mathrm{p}}} b_{j i}=1 \quad \text { and } \quad 0 \leq b_{j i} \leq 1, \quad j=1, \ldots, n_{\mathrm{p}}, \\
\forall i=1, \ldots, n_{\mathrm{s}}
\end{gathered}
$$

where $n_{\mathrm{p}}$ represents the total number of equilibrium stages in a column and $n_{\mathrm{S}}$ represents the number of streams to be distributed. Initially, the optimal design problem is solved considering that each stream is distributed to each plate in a candidate region (not necessarily composed only by adjacent equilibrium stages), subject to respective constraints of type (1). This corresponds to the solution of the continuous relaxation of the equivalent MINLP design problem. To converge the location of each stream to a single equilibrium stage, constraints of the form

$\sum_{j=1}^{n_{\mathrm{p}}} b_{j i}^{2} \geq \alpha_{i}, \quad \forall i=1, \ldots, n_{\mathrm{s}}$

are added to the previous solution, using an adjustable parameter $\alpha_{i} \in[0,1]$ relative to each stream. These simple concave constraints have the following properties:

1. When $\alpha=0$, constraint (2) is trivially satisfied for any set of values of $b_{j}$ that also satisfy (1).

2. In the limit case, when $\alpha=1$, constraint (2) can only be satisfied when one of the $b_{j}$ coefficients is unitary. Given the conservation relation (1), this property implies that the remaining $b_{j}$ coefficients must vanish; therefore, the corresponding stream has been located and converged to a single equilibrium stage. To see this, note that in this case we have simultaneously from (1) and (2):

$1=\sum_{j} b_{j}^{2} \leq \sum_{j} b_{j}=1 \quad$ and $\quad 0 \leq b_{j} \leq 1$,

which can only be satisfied when one of the coefficients is unitary and the remaining ones are null.

3. When $0 \leq \alpha_{i} \leq 1$, the fractional nature of the partition coefficients $b_{j i}$ define a threshold value $\alpha_{i, \mathrm{rl} x}$. This limit can be computed by applying Eq. (2) to the relaxed solution

$\alpha_{i, \mathrm{rlx}}=\sum_{j=1}^{n_{\mathrm{p}}} b_{j i, \mathrm{rlx}}^{2}=\sum_{j \in E_{i, \mathrm{rl} x}} b_{j i, \mathrm{rlx}}^{2}$

In this expression $E_{i, \mathrm{rl} x}$ represents the set of equilibrium stages for which the correspondent coefficients $b_{j i}$ are strictly positive, that is, the set of stages where a given stream $i$ should be directed, accordingly to the relaxed solution. For values of $\alpha_{i}<\alpha_{i, \mathrm{rl} x}$, the respective Eq. (2) becomes redundant (i.e., inactive) in the formulation.

4. A minimum amount of stream aggregation can be introduced by Eq. (2), for values of $\alpha_{i}$ that satisfy $\alpha_{i, \mathrm{rls}} \leq \alpha_{i} \leq 1$. We denote by $E_{i, \alpha}$ the set of stages where a stream $i$ is present, after solution of the optimal design problem subject to constraints (1) and (2), using a fixed value of $\alpha$. The correspondent state of aggregation of this solution, relative to stream $i$, can be measured by the maximum split fraction (supremum) observed in the set $E_{i, \alpha}$, $b_{i *}(\alpha)=\sup _{k \in E_{i, \alpha}}\left(b_{k}\right)$.

5. The state of aggregation of a solution relative to a stream $i$ varies monotonically from a minimum at $\alpha_{i}=\alpha_{i, \min }$, to a maximum for $\alpha_{i}=1$, where $b_{i *}(1)=1$, and the stream is directed entirely to one equilibrium stage. When constraint (2) is active, a simple lower bound for $b_{i^{*}}(\alpha)$ can be derived by writing

$$
\alpha=b_{*}^{2}+\sum_{j \in E_{\alpha} \backslash *} b_{j}^{2} \leq b_{*}^{2}+\sum_{j \in E_{\alpha} \backslash *} b_{j} .
$$

From (1), $b_{*}=1-\sum_{j \in E_{\alpha} \backslash *} b_{j}$, and the previous equation becomes $\alpha \leq b_{*}^{2}+1-b_{*}$, or equivalently,

$b_{*}(\alpha) \geq \frac{1+\sqrt{4 \alpha-3}}{2}$,

for $\alpha \geq 0.75$. This bound is monotone with $\alpha$, and becomes progressively tighter to the value of $b *(\alpha)$, as $\alpha \rightarrow 1$.

The present method starts with the solution of the relaxed NLP design problem, where all streams to be optimally located are distributed to each tray in their respective candidate region, subject to individual constraints of type (1). This first solution produces values of $\alpha_{i, \mathrm{rl} x}$ for each stream, which are then progressively increased towards unity in the optimization problems solved subsequently. The number of interme- 
diate steps required to full convergence to the final configuration depends on the type of stream to be converged (feed, side-stream or reflux/reboil), as well on the non-linearity of the equilibrium problem. The computational experience acquired with the examples considered indicates that, similar to the DDF approach, the premature use of large values of $\alpha$ makes the problem more sensitive to local solutions. Therefore, the effect of this parameter should be considered by cautious variation, especially on complex problems, instead of just considering directly a final solution with $\alpha=1$.

During this procedure, each NLP subproblem to be solved is initialized from the solution of the previous NLP, except in the case of the relaxed problem, which uses the values obtained during the pre-processing phase; this is considered in the next section. The warm start corresponds to an infeasible point, since increasing the value of $\alpha$ makes constraints (2) violated. Thus, especially for very large/complex columns models, a large number of infeasibilities are subsequently generated during the first iterations of the solution of each NLP subproblem, as the design variables are adapted to accommodate for constraints (2) with larger values of $\alpha$. This provides an additional argument for careful variation of the parameters $\alpha_{i}$ during the optimization procedure. Using the examples considered in the paper, and a variety of commercial NLP solvers, we found that this step was too sensitive to failures in locating a feasible solution, introducing therefore important limitations in the rates of change of $\alpha$ that could be successfully used. To avoid this type of numerical difficulties, the original NLP subproblems are instead reformulated and solved as

$$
\begin{aligned}
& \min _{x, \varepsilon} \varphi(x) \\
& \text { s.t. } f(x)-\varepsilon=0, \\
& g(x, \alpha) \leq 0 \\
& \|\varepsilon\| \leq \delta
\end{aligned}
$$

by introducing slack variables $\varepsilon$ in the model equations, and a scalar bound $\delta$ on the maximum allowed tolerance for the model equations. No slack variables were introduced in the remaining inequality constraints of the model, since in some cases, they relate to feasibility and operational constraints that need to be enforced in order to avoid failures during the evaluation of the model $f(x)$.

Similarly to the role of $\alpha$, the $\delta$ constant represents a tuning parameter in the algorithm, which can be adapted using different strategies. At the end, we must have $\alpha_{i}=1$ and $\delta=0$. However, the intermediate NLP subproblems can be solved using a variety of combinations of the values of $\alpha$ and $\delta$. Depending on the type of problem, different strategies can be used, in order to maximize the speed and robustness of the convergence to the final solution. These can be summarized as follows:

1. Fixed $\alpha$ /variable $\delta$ : This corresponds to the most aggressive, and usually is the fastest approach, since only one NLP subproblem needs to be solved for each value of $\delta$ to converge all streams to their final locations. It tends also to be more sensitive to the presence of local optima, and therefore require a closer initialization than the remaining alternatives.

2. Fixed $\delta /$ variable $\alpha$ : In this strategy, $\delta$ is maintained fixed (e.g., $\delta=10^{-6}$ ), and a sequence of NLP problems are solved until the convergence of $\alpha \rightarrow 1$ has occurred. Only after $\alpha$ has reached its final value, $\delta$ is further decreased towards 0 , if needed. In this manner, the evolution of the objective function with the minimum amount of stream aggregation can be more easily studied. This can be useful to study alternative configurations of complex columns, with several feeds and side-streams.

3. Variable $\alpha /$ variable $\delta$ : This is the most general approach, capable of potentially maximizing the numerical robustness of the method, at the cost perhaps of an increased number of NLP subproblems to be solved, relatively to the previous approaches.

The numerical performance and other merits of each of these alternatives are discussed with the examples presented in Section 5. Other modeling and initialization aspects are also critical to the successful application of this optimization strategy, in order to avoid numerical difficulties and failures during the optimization phase. These are detailed in the next section.

\section{Further modeling aspects}

\subsection{Pre-processing (initialization) phase}

The solution and optimization of distillation models corresponds, in general, to highly non-linear and non-convex problems, thus requiring careful initialization, bounding and scaling of the problem to avoid numerical difficulties. A lack of robustness can result in two kinds of problems: the first one is related with solver failures that can cause the premature end of the optimization phase; the second is related with the quality of the solutions obtained. For instance, Barttfeld et al. (2003) report that better "optimal" solutions can be obtained when a proper pre-processing phase is considered.

Pre-processing phases, used to initialize the model variables before the start of the optimization phase, have been studied in great detail by several authors. Recently, Fletcher and Morton (2000) presented a systematic procedure to overcome the difficulties mentioned, based on a limiting case of the column model, using infinite reflux or zero feed. Bruggemann and Marquardt (2001) proposed a shortcut method based on the rectification body method (RBM), providing an estimate of the minimum energy demand together with a check for feasible product specifications. Barttfeld and Aguirre (2002) developed the reversible distillation sequence model (RDSM), a method that relies on thermodynamic aspects, leading the process synthesis to energetically efficient designs. This is based on a pre-processing phase 
STEPS

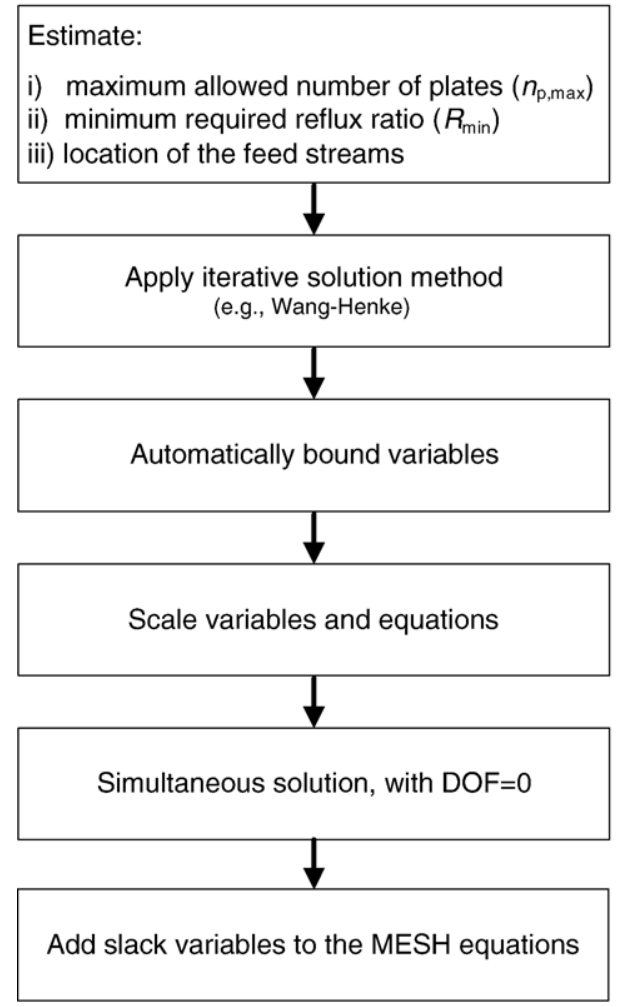

\section{OBJECTIVES}

Obtain a feasible column design closeto minimum energy demand operation.Choose the sets of candidate trays toreceive each feed.

Initialize properly all problem variables.

Fix reasonable ranges of variation.

Avoid "unbalanced" Jacobian matrix and large numerical derivatives.

Generate a feasible starting point for the optimization phase.

Avoid problems caused by infeasibilities in the early stages of the optimization phase.

Fig. 2. Schematic representation of the main steps involved in the developed pre-processing phase.

where auxiliary optimization models are solved in a sequential manner. More recently, Barttfeld et al. (2003) extended the RDSM method to azeotropic distillation. All of these approaches share several common key aspects: easy application, useful initialization of model variables and derivation of critical bounds for important operational specifications, such as minimum energy demand for each separation.The pre-processing phase used in this work, presented in Fig. 2, follows a similar path. The objective of the first phase is to generate a feasible design for each distillation column that involves its minimum energy demand, and therefore allows the derivation of a lower bound for the reflux ratio and an upper bound on the total number of equilibrium stages required. This is performed using existing shortcut methods for columns with multiple feeds and side-streams (Barnés, Hanson, \& King, 1972; Holland, 1963; Nikolaides \& Malone, 1987), taking into consideration the eventual splitting of streams throughout their candidate regions, to produce estimates for the minimum reflux ratio $\left(R_{\min }\right)$ and the number of plates $\left(n_{\mathrm{p}}\right)$ required. When necessary, these estimates can be further validated offline using a process simulator. An upper bound on the maximum number of plates of the column $\left(n_{\mathrm{p}, \max }\right)$ can be obtained in a fast trial-and-error approach by noticing when further increases on the number of plates of the column do not allow significant decreases in the reflux ratio necessary to meet the imposed operational specifications.

The second step in the pre-processing phase requires the application of one (or few) iterations of a rigorous simula- tion method, to initialize properly all model variables. The number of iterations required can be controlled by observing the residuals of the model equations. Due to its straightforward implementation and fair speed of convergence, the method of Wang-Henke (Friday \& Smith, 1964) was used with the examples considered in this study, since it can be implemented based on simple rearrangements of the massequilibrium-summation-heat balance equations (MESH) already present in the model. For columns with feed streams that present very wide boiling mixtures, more specific methods like the sum of rates (SR) or more robust methods like the simultaneous correction (SC) and the inside-out can be selected instead (Seader \& Henley, 1998).

Once in the presence of reasonable initial values for all variables, bounds for them are automatically added to the formulation, using constant scale factors appropriate to their expected range of variation. The model equations and variables are then scaled, to produce a Jacobian matrix which is approximately equilibrated. We also assume that proper care has been placed in the simplification and rearrangement of the original model equations, not only to avoid mathematical singularities, but also to produce models with simple and more linear partial derivatives.

The last step of the initialization phase consists in obtaining an exact solution of the design problem (3), relative to the configuration considered during the previous steps. In this case, the main decision variables of the problem (total number of stages, stream distribution and reflux ratio) are fixed 
to the values derived or used in the previous steps, resulting in a problem with zero degrees of freedom. Similarly, to the approach followed for the solution of the NLP subproblems, this design problem is formulated more conveniently as:

$\min _{x, \delta, \varepsilon} \delta$

s.t. $f(x)-\varepsilon=0$

$g(x, 0) \leq 0$

$\|\varepsilon\| \leq \delta$

This pre-processing phase is therefore terminated with a feasible column design, which can be subsequently used as an initial iterate in the optimization procedure.

\subsection{Selecting the total number of trays}

The solution produced during the pre-processing phase is convenient not only because it corresponds to a feasible column design but also because it allows the selection of the total number of plates, based on the use of constraints of type (1) and (2) as well, using the approaches developed for this purpose in the context of discrete optimization. Presently, two main schemes have been reported for the elimination of trays during the optimization of a distillation unit. The first one was introduced by Viswanathan and Grossmann (1993) and uses a variable location of the reflux or reboil streams to eliminate one of the streams present in certain trays. The second scheme, presented recently by Barttfeld et al. (2003), is based on the variable location of the heat exchanging equipment (condensers/reboilers). These authors conclude that this approach allows a more efficient MINLP representation of the problem.

The examples considered in this paper were solved using the classical variable reflux and/or reboil scheme, although both approaches can be readily implemented. Fig. 3 illustrates the different alternatives to reduce the initial number of plates $\left(n_{\mathrm{p}, \max }\right)$, using the variable location of the top and bottom streams. In case (a), the reflux is split between the candidate trays located immediately below the condenser. During the optimization procedure, all plates located above the selected entrance for the reflux will lose their liquid phase. In the case (b) of Fig. 3, the reboil is distributed by the candidate trays located immediately above the reboiler. Here all trays located below the selected optimal entrance for the reboil stream will lose their vapor stream. In case (c) of Fig. 3, both of the previous situations are applicable.

The most appropriate superstructure for a particular example depends on the relative location of the feed streams in the column. The variable reflux approach tends to be more efficient when the feed streams are located close to the bottom of the column, while the variable reboil approach is advised for units where the location of the feed streams are closer to the top; the variable reflux and reboil is the most flexible approach, at the cost of having to locate one additional stream relatively to each of the previous cases. Although not illustrated in Fig. 3, it should be emphasized that the possible overlap of the candidate regions for the location of the feed, side-streams and reflux/reboil does not introduce special numerical difficulties in the formulation, provided that special care is used to count the total number of effective equilibrium stages, for use in the objective function.

When a plate loses one of the phases, the corresponding liquid-vapor equilibrium relations might not remain applicable, and the remaining MESH equations for that stage can be greatly simplified. GDP-based strategies use logical constraints to model these special cases, in order to avoid possible numerical difficulties. Using a continuous strategy, Lang and Biegler (2002) address this potential problem by adding complementary constraints to the original problem, taking advantage of a previously developed smoothing function approach to model the loss of phases during the optimization of a flash unit (Gopal \& Biegler, 1999). Their main idea is to rewrite the equilibrium balance equations introducing slack variables that assume different values accordingly to the complementary equations. These last ones are modeled by smoothing functions in the Lang and Biegler (2002) ap-

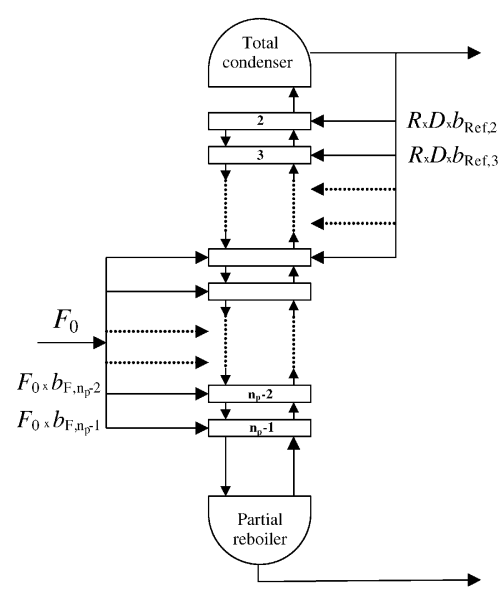

(a)

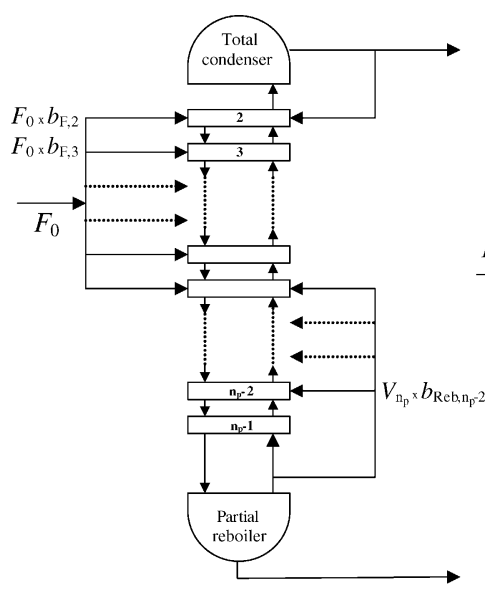

(b)

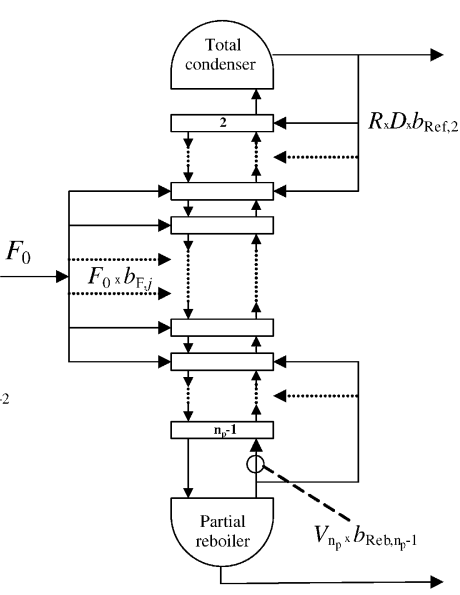

(c)

Fig. 3. Available approaches for tray reduction: (a) variable reflux, (b) variable reboil and (c) simultaneous variable reflux and reboil. 
Table 1

Optimal design of a binary distillation unit for ciclohexanol/water

\begin{tabular}{cllllll}
\hline Plate & $L_{j}$ & $V_{j}$ & $T_{j}$ & $x_{1, j}$ & $y_{1, j}$ & $K_{1, j}$ \\
\hline cond. & 0.156 & 0.000 & 426.773 & 0.542 & 0.833 & 1.537 \\
2 & 0.000 & 0.672 & 442.052 & 0.277 & 0.542 & 1.954 \\
3 & 0.000 & 0.672 & 442.052 & 0.277 & 0.542 & 1.954 \\
4 & 0.152 & 0.672 & 442.052 & 0.277 & 0.542 & 1.954 \\
5 & 0.151 & 0.668 & 444.289 & 0.240 & 0.482 & 2.005 \\
6 & 2.333 & 0.667 & 444.582 & 0.235 & 0.473 & 2.011 \\
7 & 2.332 & 0.750 & 445.200 & 0.225 & 0.455 & 2.024 \\
8 & 2.329 & 0.748 & 446.258 & 0.207 & 0.424 & 2.045 \\
9 & 2.324 & 0.745 & 447.988 & 0.178 & 0.369 & 2.077 \\
10 & 2.324 & 0.000 & 447.988 & 0.178 & 0.369 & 2.077 \\
11 & 2.324 & 0.000 & 447.988 & 0.178 & 0.369 & 2.077 \\
reb. & 1.584 & 0.740 & 450.619 & 0.131 & 0.277 & 2.112 \\
\hline
\end{tabular}

The location of the feed, reflux and reboil streams are, respectively, Plates 6, 4 and 9. Additional problem data: $F=2.1 \mathrm{kmol} / \mathrm{h}, x_{\mathrm{F}}=\{0.232,0.768\}$, $D=0.516 \mathrm{kmol} / \mathrm{h}, R=0.3023$. The feed is slightly subcooled, and the equilibrium data are based on the UNIFAC method.

proach, and addressed as MPEC problems by Raghunathan and Biegler (2003).

The need to use these complementary constraints is alleviated if a negligible pressure drop (reasonable for most columns operating near the atmospheric pressure) and no heat losses are assumed for the region of the column where one of the phases disappears. In this case, the original MESH equations are still verified throughout this region, although with zero flows and equilibrium compositions that might correspond to fictitious (although physically viable) phases. This is illustrated with the problem presented in Table 1. As can be observed, the disappearance of a physical phase is associated with the beginning of a region of constant composition and zero flow for that phase. In this manner, the original formulation can still be used. The examples considered in this paper rely on the above assumptions. In more complex situations, the use of one of the previous modifications is recommended.

\section{Mathematical formulation}

Eqs. (4)-(9) represent the complete formulation for the economical optimization of a distillation unit, using the approach of Fig. 1(b), for:

- a column with a total condenser and a partial reboiler;

- non-ideal behavior of the liquid and vapor phases;

- constant pressure along the column;

- variable reflux and reboil approach to reduce the number of plates;

- liquid and vapor side-streams allowed to enter all equilibrium stages, with the exception of the condenser and the reboiler.

Properties correlations

$$
\begin{aligned}
H_{\mathrm{L}, j} & =f\left(x_{i j}, T_{j}\right), \\
H_{\mathrm{V}, j} & =f\left(y_{i j}, T_{j}\right), K_{i j}=f\left(x_{i j}, y_{i j}, T_{j}\right) ; \\
i & =1, \ldots, n_{\mathrm{c}} ; \quad j=1, \ldots, n_{\mathrm{p}}
\end{aligned}
$$

Phase compositions summation

$\sum_{i}^{n_{\mathrm{c}}} x_{i j}=1 \quad$ and $\quad \sum_{i}^{n_{\mathrm{c}}} y_{i j}=1, \quad j=1, \ldots, n_{\mathrm{p}}$

Phase equilibrium balances

$y_{i j}=K_{i j} x_{i j}-\varepsilon_{\mathrm{EQ}, i j} ; \quad i=1, \ldots, n_{\mathrm{c}} ; \quad j=1, \ldots, n_{\mathrm{p}}$

Mass and energy balances

Condenser $\left(i=1, \ldots, n_{\mathrm{c}} ; j=1\right)$

$$
\begin{aligned}
& V_{j+1}=L_{j}\left(1+\frac{1}{R}\right)-\varepsilon_{\mathrm{BMT}, j} \\
& V_{j+1} y_{i, j+1}=L_{j}\left(1+\frac{1}{R}\right) x_{i, j}-\varepsilon_{\mathrm{BMP}, i, j} \\
& Q_{\mathrm{C}}+V_{j+1} H_{\mathrm{V}, j+1}=L_{j}\left(1+\frac{1}{R}\right) H_{\mathrm{L}, j}-\varepsilon_{\mathrm{BE}, j} \\
& \text { Column }\left(i=1, \ldots, n_{\mathrm{c}} ; j=2, \ldots, n_{\mathrm{p}}-1\right)^{1} \\
& L_{j-1}+V_{j+1}+F_{0} b_{\mathrm{F}, j}+R D b_{\mathrm{Ref}, j}+V_{n_{\mathrm{p}}} b_{\mathrm{Reb}, j} \\
& \quad=\left(L_{j}+U_{0} b_{\mathrm{LS}, j}+V_{j}+W_{j}\right)-\varepsilon_{\mathrm{BMT}, j} \\
& L_{j-1} x_{i, j-1}+V_{j+1} y_{i, j+1}+F_{0} b_{\mathrm{F}, j} x_{\mathrm{F}, i} \\
& \quad+R D b_{\mathrm{Ref}, j} x_{i, 1}+V_{n_{\mathrm{p}}} b_{\mathrm{Reb}, j} y_{i, n_{\mathrm{p}}} \\
& =\left(L_{j}+U_{0} b_{\mathrm{LS}, j}\right) x_{i, j}+\left(V_{j}+W_{0} b_{\mathrm{VS}, j}\right) y_{i, j}-\varepsilon_{\mathrm{BMP}, i, j}
\end{aligned}
$$

$$
\begin{aligned}
& L_{j-1} H_{\mathrm{L}, j-1}+V_{j+1} H_{\mathrm{V}, j+1}+F_{0} b_{\mathrm{F}, j} H_{\mathrm{F}} \\
& \quad+R D b_{\mathrm{Ref}, j} H_{\mathrm{L}, 1}+V_{n_{\mathrm{p}}} b_{\mathrm{Reb}, j} H_{\mathrm{V}, n_{\mathrm{p}}} \\
& \quad\left(L_{j}+U_{0} b_{\mathrm{LS}, j}\right) H_{\mathrm{L}, j}+\left(V_{j}+W_{0} b_{\mathrm{VS}, j}\right) H_{\mathrm{V}, j}-\varepsilon_{\mathrm{BE}, j}
\end{aligned}
$$

Reboiler $\left(i=1, \ldots, n_{\mathrm{c}} ; j=n_{\mathrm{p}}\right)$

$$
\begin{aligned}
& L_{j-1}=L_{j}+V_{j}-\varepsilon_{\mathrm{BMT}, j} \\
& L_{j-1} x_{i, j-1}=L_{j} x_{i, j}+V_{j} y_{i, j}-\varepsilon_{\mathrm{BMP}, i, j} \\
& Q_{\mathrm{R}}+L_{j-1} H_{\mathrm{L}, j-1}=L_{j} H_{\mathrm{L}, j}+V_{j} H_{\mathrm{V}, j}-\varepsilon_{\mathrm{BE}, j}
\end{aligned}
$$

Auxiliary expressions

\footnotetext{
${ }^{1}$ For $j=2$, terms $L_{j-1}, L_{j-1} x_{i, j-1}$ and $L_{j-1} H_{\mathrm{L}, j-1}$ disappear, respectively, in the left side of the equations representing the total mass balance, partial mass balance and energy balance. For $j=n_{\mathrm{p}}-1$, terms $V_{j+1}, V_{j+1} y_{i, j+1}$ and $V_{j+1} H_{\mathrm{V}_{j}+1}$ disappear, respectively, in the same equations earlier described.
} 
Split fractions summation

$$
\begin{aligned}
& \sum_{j=1}^{n_{\mathrm{p}}} b_{\mathrm{F}, j}=1, \quad \sum_{j=1}^{n_{\mathrm{p}}} b_{\mathrm{Ref}, j}=1, \quad \sum_{j=1}^{n_{\mathrm{p}}} b_{\mathrm{Reb}, j}=1, \\
& \sum_{j=1}^{n_{\mathrm{p}}} b_{\mathrm{LS}, j}=1, \quad \sum_{j=1}^{n_{\mathrm{p}}} b_{\mathrm{VS}, j}=1
\end{aligned}
$$

$0 \leq b_{i j} \leq 1, \quad i=\{\mathrm{F}$, Ref, Reb, LS, VS $\}$

$j=1, \ldots, n_{\mathrm{p}}$

Feed and product streams convergence

$$
\begin{aligned}
& \sum_{j=1}^{n_{\mathrm{p}}} b_{\mathrm{F}, j}^{2} \geq \alpha_{\mathrm{F}}, \quad \sum_{j=1}^{n_{\mathrm{p}}} b_{\mathrm{Ref}, j}^{2} \geq \alpha_{\mathrm{Ref}}, \\
& \sum_{j=1}^{n_{\mathrm{p}}} b_{\mathrm{Reb}, j}^{2} \geq \alpha_{\mathrm{Reb}}, \quad \sum_{j=1}^{n_{\mathrm{p}}} b_{\mathrm{LS}, j}^{2} \geq \alpha_{\mathrm{LS}}, \\
& \sum_{j=1}^{n_{\mathrm{p}}} b_{\mathrm{VS}, j}^{2} \geq \alpha_{\mathrm{VS}}
\end{aligned}
$$

$0 \leq \alpha_{i} \leq 1, \quad i=\{\mathrm{F}$, Ref, Reb, LS, VS $\}$

Slack variable constraints

$$
\begin{aligned}
& \left\|\varepsilon_{\mathrm{BMT}, j}\right\| \leq \delta, \quad\left\|\varepsilon_{\mathrm{BMP}, i, j}\right\| \leq \delta, \quad\left\|\varepsilon_{\mathrm{EQ}, i, j}\right\| \leq \delta, \\
& \left\|\varepsilon_{\mathrm{BE}, j}\right\| \leq \delta ; \quad i=1, \ldots, n_{\mathrm{c}} ; \quad j=1, \ldots, n_{\mathrm{p}}
\end{aligned}
$$

Operational specifications in side-streams (purities)

$$
\begin{aligned}
& \frac{\sum_{j}^{n_{\mathrm{p}}}\left(b_{\mathrm{LS}, j} x_{i, j} U_{0}\right)}{\sum_{j}^{n_{\mathrm{p}}}\left(\sum_{i}^{n_{\mathrm{c}}} b_{\mathrm{LS}, j} x_{i, j} U_{0}\right)} \frac{\geq}{<} S_{\mathrm{LS}, i}, \quad i=1, \ldots, n_{\mathrm{c}} \\
& \frac{\sum_{j}^{n_{\mathrm{p}}}\left(b_{\mathrm{VS}, j} y_{i, j} W_{0}\right)}{\sum_{j}^{n_{\mathrm{p}}}\left(\sum_{i}^{n_{\mathrm{c}}} b_{\mathrm{VS}, j} y_{i, j} W_{0}\right)} \frac{>}{<} S_{\mathrm{VS}, i}, \quad i=1, \ldots, n_{\mathrm{c}}
\end{aligned}
$$

Cost functions

Fixed column costs (shell and internals)

$d=f\left(V_{j}, T_{j}\right) \quad$ and $\quad h=f\left(b_{\mathrm{Ref}, j}, b_{\mathrm{Reb}, j}\right)$

$C_{\mathrm{SI}}=f(d, h)$

Fixed exchanger costs (condenser/reboiler)

$$
\begin{aligned}
& A_{\mathrm{C}}=f\left(T_{1}, Q_{\mathrm{C}}\right) \text { and } A_{\mathrm{R}}=f\left(T_{n_{\mathrm{p}}}, Q_{\mathrm{R}}\right) \\
& C_{\mathrm{HE}}=f\left(A_{\mathrm{C}}, A_{\mathrm{R}}\right)
\end{aligned}
$$

Operational heat exchanger costs

$$
\begin{aligned}
& C_{\mathrm{CU}}=f\left(Q_{\mathrm{C}}, T_{1}\right) \\
& C_{\mathrm{HU}}=f\left(Q_{\mathrm{R}}, T_{n_{\mathrm{p}}}\right)
\end{aligned}
$$

Total costs

$$
C_{\mathrm{T}}=C_{\mathrm{SI}}+C_{\mathrm{HE}}+C_{\mathrm{CU}}+C_{\mathrm{HU}}
$$

The objective of the problem is to minimize the total annualized cost $\left(C_{\mathrm{T}}\right)$ that involves both equipment and utilities costs:

$$
\begin{array}{cc}
\min _{b_{\mathrm{F}, j}, b_{\mathrm{Ref}, j}, b_{\mathrm{Reb}, j}, b_{\mathrm{LS}, j}, b_{\mathrm{VS}, j}, R} & C_{\mathrm{T}} \\
\text { s.t. } & (4)-(9)
\end{array}
$$

\section{Application examples}

Several case studies are considered to illustrate the performance of the continuous optimization strategy developed. Most of these examples are taken from the purification phase of the aniline production process, by hydrogenation of nitrobenzene in the liquid phase. This industrial process involves the separation of 10 components, with some of them present in vestigial concentrations. The first example considers an existing industrial unit (with fixed $n_{\mathrm{p}}$ ), to demonstrate the gains that can be achieved in the optimization of complex columns, by allowing the split of the feed between non-consecutive trays in the final configuration. This is an important advantage over the previous continuous formulation.

The second example involves three existing units that assure the aniline purification phase in the process implemented by Quimigal, S.A. This illustrates the effectiveness of the adopted pre-processing phase in avoiding numerical difficulties in large-scale problems. Additionally, it also reveals the large economical benefits that can be obtained by the simultaneous optimization of existing sets of distillation columns.

The third refers to an extractive distillation column, drawn from the winner solution of the EURECHA student contest competition in 2004 (EURECHA, 2004). It presents another situation where feed splitting in the final configuration is advantageous, and therefore must be accounted during the project of a new unit ( $n_{\mathrm{p}}$ variable).

The final example refers to the design of a new conventional unit. Contrarily to the optimization of complex columns, this example shows no significant advantage in allowing the feed to be split in the final configuration of simple columns. In all cases, the UNIFAC group contribution method was used to describe the vapor-liquid equilibria. The resulting mathematical models were formulated in GAMS (Brooke et al., 1998), and solved using CONOPT III, in a 2.6 GHz Pentium IV computer.

\subsection{Example 1}

This example considers a distillation unit with 10 components $(\mathrm{c}-01, \ldots, \mathrm{c}-10)$, from the purification of aniline. Here, component $\mathrm{c}-06$ is the required product, components $\mathrm{c}-01$, ..., c-05 are "light" parasites and components c-07, . , c-10 are "heavy" parasites, accordingly to the relative volatilities 
Table 2

Relative fractions and volatilities of the components present in the feed streams of the distillation unit discussed in Section 5.1

\begin{tabular}{lrrrrrrrrrrr}
\hline \multicolumn{1}{l}{ Components } \\
\cline { 2 - 9 } & $\mathrm{c}-01$ & $\mathrm{c}-02$ & $\mathrm{c}-03$ & $\mathrm{c}-04$ & $\mathrm{c}-05$ & $\mathrm{c}-06$ & $\mathrm{c}-07$ & $\mathrm{c}-08$ & $\mathrm{c}-09$ & $\mathrm{c}-10$ \\
\hline Relative fractions & $<0.01$ & 37.19 & 8.21 & 12.00 & 37.58 & NA & $<0.01$ & 0.31 & 4.44 & 0.27 \\
S1 & $<0.01$ & 4.05 & 3.72 & 9.03 & 26.74 & NA & $<0.01$ & 0.82 & 40.30 & 15.32 \\
S2 & $<0.01$ & 2.07 & 0.64 & 2.12 & 1.45 & NA & $<0.01$ & 2.03 & 52.19 & 38.74 \\
S3 & 57.82 & 21.24 & 4.91 & 3.42 & 3.27 & 1.00 & 0.97 & 0.92 & 0.10 & 0.04 \\
Relative mean volatilities & & & & & & & & &
\end{tabular}

presented in Table 2. This column has three feeds with compositions also presented in Table 2: stream S1, rich in four components lighter than the desired product, stream S3 rich in two heavy components, and stream $\mathrm{S} 2$ rich both in lower and upper boiling point components. The required purity of the middle boiling point component in the liquid side-stream (S5) is greater than $99.96 \%$.

Estimates for the minimum required reflux ratio for this separation were made using the pre-processing method described earlier, considering $n_{\mathrm{p}}=9$ (fixed). After this, the feed streams were split among the respective candidate trays: stream S1 equally split between Plates 2 and 5, streams S2 and S3 divided between Plates $n_{\mathrm{p}}-1$ and $n_{\mathrm{p}}-6$, and stream S5 between Plate 5 and Plate $n_{\mathrm{p}}-6$. This selection of sets of candidate trays was based on the information drawn from the pre-processing phase, regarding the estimates for the most favorable regions of the column to receive each of the feeds.

Table 3 summarizes the convergence data for the optimization of this unit. This study involved a mathematical model with approximately 7000 equations/variables, and a CPU time of $12 \mathrm{~s}$ for the pre-processing phase, excluding the first step, which was done separately. It can be noticed that with a fixed $\alpha /$ variable $\delta$ strategy, the maximum number of infeasibilities observed during the sequential steps of the optimization procedure decreased, together with the CPU time necessary for the solution of the problem. However, an important advantage of the strategy with variable $\alpha$ is that interesting economic configurations can be investigated, as shown in Fig. 4. In this study, the locations of the streams $\mathrm{S} 1, \mathrm{~S} 3$ and S5 remained fixed, but $\alpha_{\mathrm{F}}$ was relaxed to 0 . This allowed the identification of a configuration with a smaller reflux ratio, when $\mathrm{S} 2$ is allowed to be split between Plate 5 $(37 \%)$ and Plate $8(63 \%)$. The corresponding optimal configurations found are presented in Fig. 5.

Table 3

Convergence data obtained for the optimization of the distillation unit considered in Section $5.1\left(\alpha=\alpha_{\mathrm{LS}}=\alpha_{\mathrm{F}}\right)$

\begin{tabular}{lll}
\hline & \multicolumn{2}{l}{ Optimization phase } \\
\cline { 2 - 3 } & $\begin{array}{l}\text { Infeasibilities } \\
\text { (maximum number) }\end{array}$ & CPU time (s) \\
\hline Fixed $\delta$, variable $\alpha$ & 238 & 41 \\
Fixed $\alpha$, variable $\delta$ & 115 & 29 \\
\hline
\end{tabular}

\subsection{Example 2}

The second industrial case study involves the simultaneous optimization of a set of three distillation columns, represented in Fig. 6. It comprises four liquid feeds (S1, S5, S9 and S10), one vapor feed (S4) and one liquid side-stream (S7). The objective is to minimize the heat consumption in the arrangement, with fixed $n_{\mathrm{p}}$, since these are existing columns. The composition of the independent feed stream and the required product specification in S7 are shown in Table 4.The pre-processing phase for this problem included the application of the previous initialization methodology described in Section 3.1 individually to each column, combined with a simultaneous iterative strategy based on the use of constant split fractions, solution of linear mass balances, and recalculation of the individual split fractions (Biegler, Grossmann, \& Westerberg, 1997), to generate a feasible starting point for the set of columns. The main results are presented in Tables 5 and 6, both for the individual optimization of each column, and the simultaneous optimization of the arrangement. In Table 5, a value of -1 means that the optimal location of the stream is now in the equilibrium stage above the one used in the existing industrial configuration. As can be observed, a simultaneous optimization procedure provides significant economical benefits relative both to the present industrial configuration and to the separate optimization of each unit. The individual optimization of column $\mathrm{C}$ was not considered, because the flow rates involved in this unit were considered too small to generate significant economies.

This study involved the solution of a model with approximately 18,000 equations and variables; $245 \mathrm{~s}$ were spent in the pre-processing phase, and $157 \mathrm{~s}$ were necessary to obtain the relaxed solution of the problem. The remaining convergence data are presented in Table 7. It is again possible to notice that with a variable $\delta$ approach the total CPU time and the maximum number of infeasibilities obtained is lower than in a situation where $\delta$ remains fixed during the entire procedure. The use of sequential steps in $\alpha$ was not adequate with this example, since the relaxed solution already presented values for these parameters very close to the unity: all streams entered in single locations, except one that was split $97 \%$ to one plate and the remaining $3 \%$ to another non-contiguous plate. 


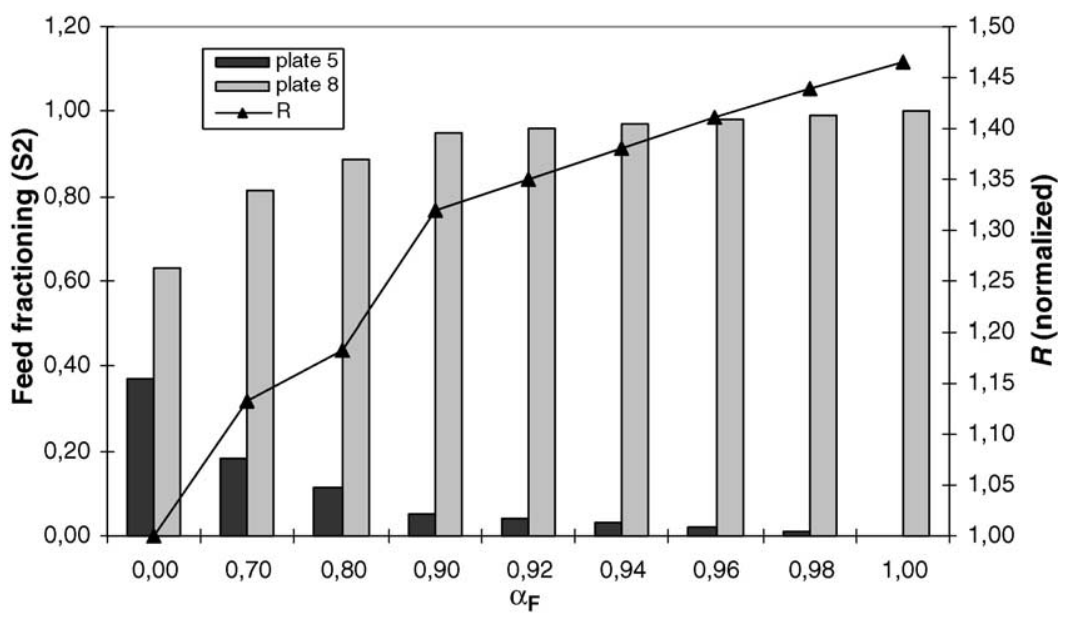

Fig. 4. Effect of the adjustable parameter $\alpha_{\mathrm{F}}$ on the fractioning of stream S2 and required reflux ratio (Section 5.1).

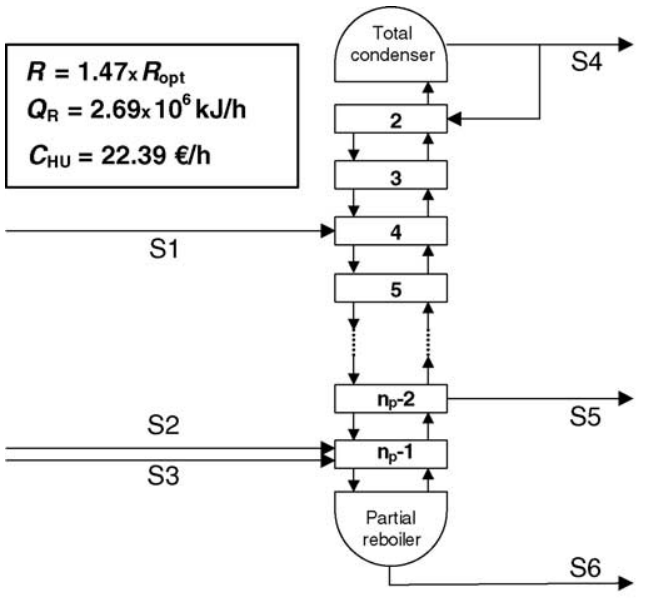

(a)

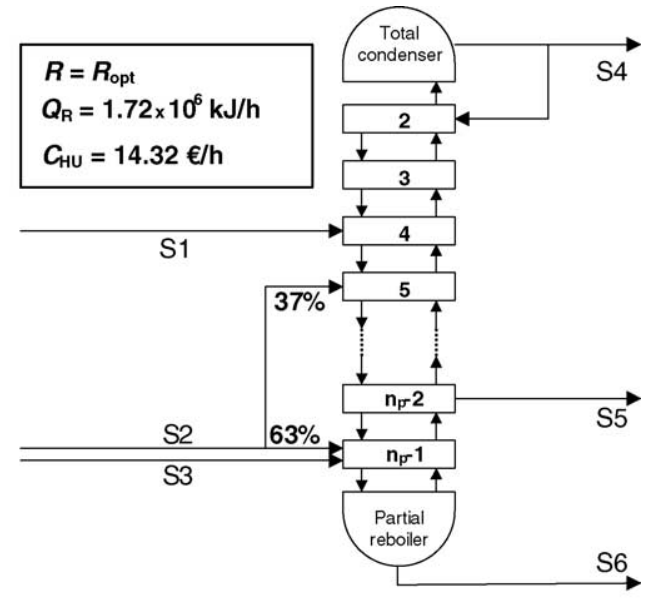

(b)

Fig. 5. Best configurations obtained by (a) DDF and (b) split fraction strategy (Section 5.1).

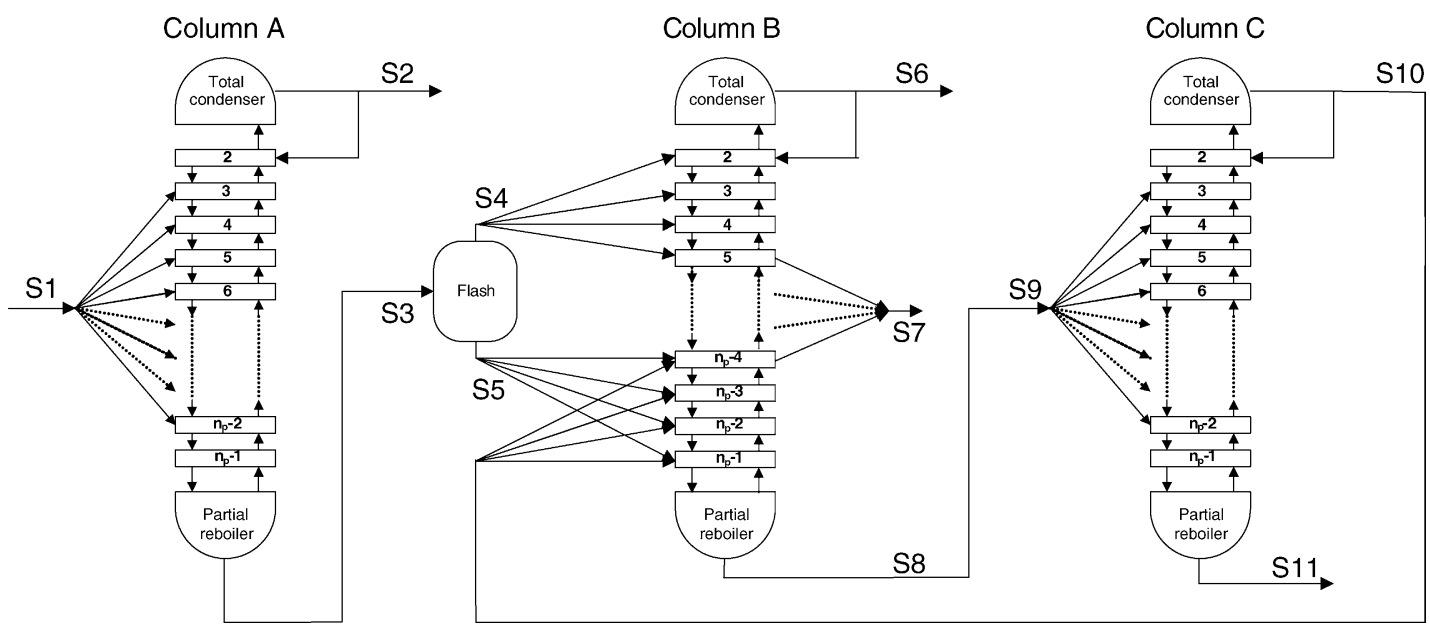

Fig. 6. Schematic representation of the stream splitting strategy for the set of distillation units involved in Section 5.2. 
Table 4

Feed and product side-stream specifications for the set of distillation units considered in Section 5.2

\begin{tabular}{lllllllllll}
\hline Components & $\mathrm{c}-01$ & $\mathrm{c}-02$ & $\mathrm{c}-03$ & $\mathrm{c}-04$ & $\mathrm{c}-05$ & $\mathrm{c}-06$ & $\mathrm{c}-07$ & $\mathrm{c}-08$ & $\mathrm{c}-09$ & $\mathrm{c}-10$ \\
\hline Relative fraction in S1 & 0.68 & 89.72 & 4.30 & 3.11 & 0.94 & $\mathrm{NA}$ & 0.03 & 0.49 & 0.71 & 0.02 \\
Absolute fraction required in S7 & $<50 \mathrm{ppm}$ & $<300 \mathrm{ppm}$ & $<50 \mathrm{ppm}$ & $<250 \mathrm{ppm}$ & $<300 \mathrm{ppm}$ & $>99.97 \%$ & $<50 \mathrm{ppm}$ & $<10 \mathrm{ppm}$ & $<250 \mathrm{ppm}$ & $<50 \mathrm{ppm}$ \\
\hline
\end{tabular}

Table 5

Plate displacement, relative to the present industrial configuration, obtained in the individual and simultaneous optimization of the distillation units (Section 5.2)

\begin{tabular}{llll}
\hline Streams & \multicolumn{2}{l}{ Plate displacement } & \\
\cline { 2 - 4 } & $\begin{array}{l}\text { Column A } \\
\text { optimization }\end{array}$ & $\begin{array}{l}\text { Column B } \\
\text { optimization }\end{array}$ & $\begin{array}{l}\text { Simultaneous } \\
\text { optimization }\end{array}$ \\
\hline S1 & -1 & NA & -1 \\
S4 & NA & -2 & 0 \\
S5 & NA & -1 & -1 \\
S7 & NA & -1 & -1 \\
S9 & NA & NA & +1 \\
S10 & NA & -3 & -3 \\
\hline
\end{tabular}

Table 6

Reflux ratio decrease and total utility consumption savings, obtained in the individual and global optimization of the distillation units (Section 5.2)

\begin{tabular}{llll}
\hline & $\begin{array}{l}\text { Column A } \\
\text { optimization }\end{array}$ & $\begin{array}{l}\text { Column B } \\
\text { optimization }\end{array}$ & $\begin{array}{l}\text { Simultaneous } \\
\text { optimization }\end{array}$ \\
\hline $\begin{array}{l}\text { Decrease of } R \\
(A / B / C)(\%)\end{array}$ & $80 / \mathrm{NA} / \mathrm{NA}$ & $\mathrm{NA} / 13 / \mathrm{NA}$ & $32 / 13 / 49$ \\
$\begin{array}{c}\text { Utilities savings } \\
(\times 1000 € / \text { year })\end{array}$ & 30.9 & 60.9 & 74.3 \\
\hline
\end{tabular}

Table 7

Convergence data obtained for the optimization of the distillation units discussed in Section $5.2\left(\alpha=\alpha_{\mathrm{LS}}=\alpha_{\mathrm{F}}\right)$

\begin{tabular}{lll}
\hline & \multicolumn{2}{l}{ Optimization phase } \\
\cline { 2 - 3 } & $\begin{array}{l}\text { Infeasibilities } \\
\text { (maximum number) }\end{array}$ & CPU time (s) \\
\hline $\begin{array}{l}\text { Fixed } \alpha, \text { fixed } \delta \\
\left(\delta=1 \times 10^{-6}\right)\end{array}$ & 2911 & 785 \\
$\begin{array}{l}\text { Fixed } \alpha, \text { variable } \delta \\
\left(\delta=1 \times 10^{-5}, 5 \times 10^{-6},\right.\end{array}$ & 1537 & 567 \\
$\left.1 \times 10^{-6}\right)$ & & \\
\hline
\end{tabular}

\subsection{Example 3}

The next example involves the optimization of an extractive column represented in Fig. 7. This unit has two feeds-stream S1, where a mixture of three organic components and water is present, and from which ethyl acetate must be recovered; stream S2, mainly composed by an extractive agent (1,4-butanediol) used to break the existing azeotropes that prevent a direct separation. The compositions of the feed streams and the ethyl acetate specifications in the distillate stream are also included in Fig. 7. Due to the highly

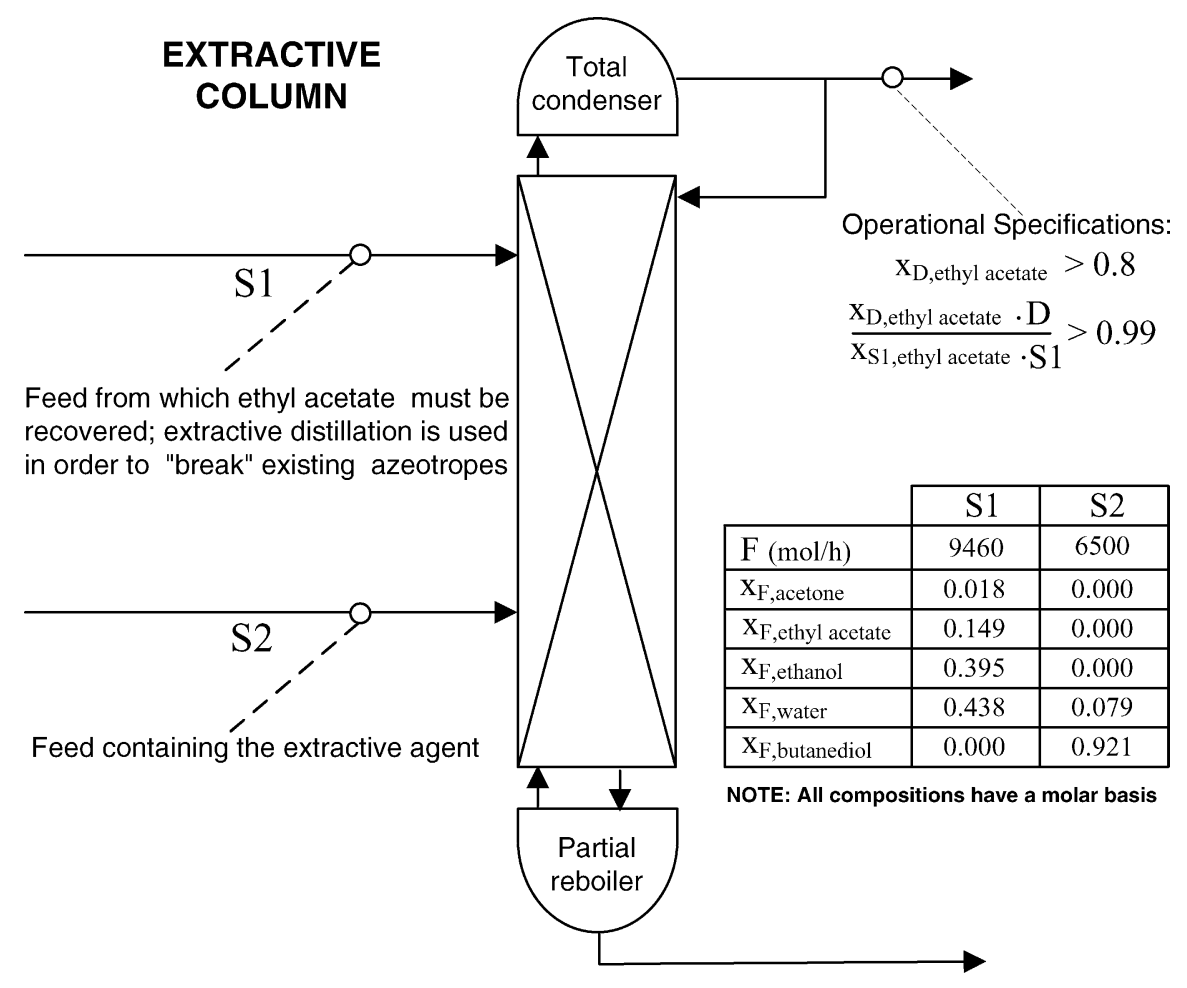

Fig. 7. Schematic representation of the extractive distillation column discussed in Section 5.3. 
Table 8

Convergence data obtained for the optimization of the distillation unit discussed in Section $5.3\left(\alpha=\alpha_{\mathrm{F}}=\alpha_{\mathrm{Reb}}\right)$

\begin{tabular}{llc}
\hline & \multicolumn{2}{l}{ Optimization phase } \\
\cline { 2 - 3 } & $\begin{array}{l}\text { Infeasibilities } \\
\text { (maximum number) }\end{array}$ & CPU time (s) \\
\hline Fixed $\delta$, variable $\alpha$ & 1542 & 104 \\
Fixed $\alpha$, variable $\delta$ & 383 & 39 \\
\hline
\end{tabular}

non-ideal LVE exhibited by the mixture considered (four binary and a ternary azeotrope are present), the UNIFAC method was selected. The objective of this example is to design a new unit with minimum annualized total cost. According to information drawn from preliminary calculations, the following decisions were made:

- The initial number of plates was set to 45 , generating a problem with approximately 9000 equations/variables.

- A fixed reflux/variable reboil scheme was selected in order to reduce this maximum number of trays.

- The candidate region to receive S1 included the Plates between 10 and 30, for stream S2 those between Plates 2 and 44, and those between Plates 20 and 44 for the reboil stream.

The economical data for this example were taken from the design literature (Turton, Bailie, Whiting, \& Shaeiwitz, 1998). In order to preserve the continuous formulation of the optimization problem, the discrete economical information was fitted to continuous functions between certain limits that determined valid lower and upper bounds on the equipment dimensions, to be considered during the optimization.

Under these conditions, $105 \mathrm{~s}$ were spent in the preprocessing phase and $103 \mathrm{~s}$ were necessary to obtain the relaxed solution of the problem; this last one presented the feed and reboil streams split in the following manner: $\mathrm{S} 1-100 \%$ in Plate 18, S2-75\% in Plate 2 and $25 \%$ in Plate 26, reboil- $35 \%$ in Plate 25 and $65 \%$ in Plate 26. The convergence data for this example are presented in Table 8, relatively to the solution where all streams are located in single equilibrium stages. Again, using a variable $\delta$ approach, the total CPU time and the maximum number of

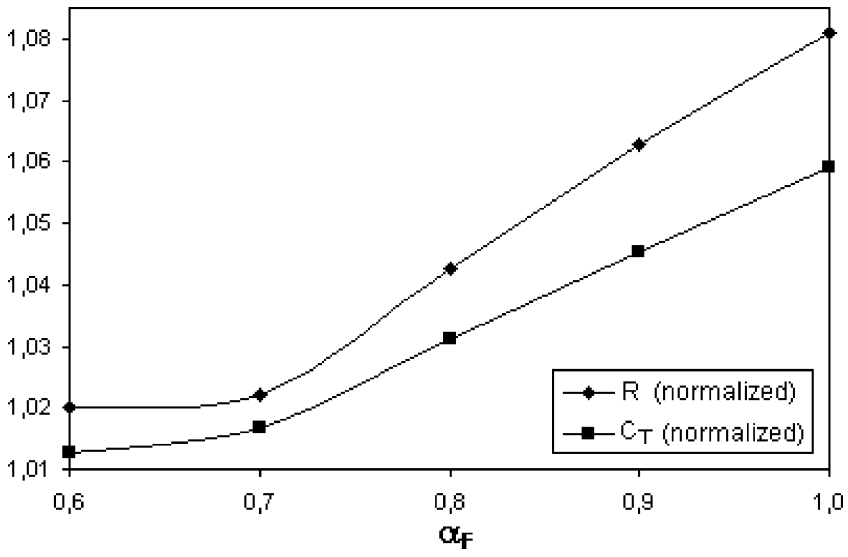

Fig. 8. Variation of required reflux ratio and total annualized costs with the adjustable parameter $\alpha_{\mathrm{F}}$ (Section 5.3).

Table 9

Optimal configurations of the distillation unit discussed in Section 5.3

\begin{tabular}{lll}
\hline & $\begin{array}{l}\text { Aggregated } \\
\text { solution }\end{array}$ & $\begin{array}{l}\text { Fractioned } \\
\text { solution }\end{array}$ \\
\hline Total number of plates & 27 & 27 \\
Location of S1 & $100 \%$ on Plate 18 & $100 \%$ on Plate 18 \\
Location of S2 & $100 \%$ on Plate 2 & $75 \%$ on Plate 2; \\
$R$ & & $25 \%$ on Plate 26 \\
$C_{\mathrm{T}}(\times 1000 € /$ year $)$ & 5.31 & 4.95 \\
\hline
\end{tabular}

infeasibilities obtained is lower than in a situation where $\delta$ remains fixed during the entire procedure.

The variable $\delta /$ fixed $\alpha$ and fixed $\alpha /$ variable $\delta$ approaches were again used to select possible final configurations for this unit. Fig. 8 presents normalized values of $C_{\mathrm{T}}$ and $R$ as a function of the parameter $\alpha_{\mathrm{F}}$. As can be seen, it is advantageous to allow one of the feeds (S2) to be split among non-contiguous plates, because the operational costs of the column become lower. Table 9 compares the main characteristics of the optimal designs obtained in both cases. As can be observed, the low CPU times required in this example are justified by the proximity of the relaxed solution to the final optimal configurations.

Table 10

Convergence data obtained for the optimization of the distillation unit discussed in Section $5.4\left(\alpha=\alpha_{\mathrm{F}}=\alpha_{\mathrm{Reb}}\right)$

\begin{tabular}{|c|c|c|c|c|}
\hline & & \multicolumn{3}{|c|}{ Optimization } \\
\hline & & $\alpha=0.8$ & $\alpha=0.9$ & $\alpha=1.0$ \\
\hline $\begin{array}{l}\text { Variable } \alpha, \text { fixed } \delta \\
\left(\delta=1 \times 10^{-6}\right)\end{array}$ & $\begin{array}{l}\text { Infeasibilities (maximum number) } \\
\text { CPU time (s) }\end{array}$ & $\begin{array}{r}1209 \\
153\end{array}$ & $\begin{array}{r}1205 \\
141\end{array}$ & $\begin{array}{r}1509 \\
156\end{array}$ \\
\hline $\begin{array}{l}\text { Variable } \alpha \text {, variable } \delta \\
\left(\delta=1 \times 10^{-5}, 5 \times 10^{-6}, 1 \times 10^{-6}\right)\end{array}$ & $\begin{array}{l}\text { Infeasibilities (maximum number) } \\
\text { CPU time (s) }\end{array}$ & $\begin{array}{l}896 \\
217\end{array}$ & $\begin{array}{l}875 \\
235\end{array}$ & $\begin{array}{l}827 \\
201\end{array}$ \\
\hline $\begin{array}{l}\text { Fixed } \alpha \text {, variable } \delta \\
\left(\delta=1 \times 10^{-5}, 1 \times 10^{-6}, 1 \times 10^{-6}\right)\end{array}$ & $\begin{array}{l}\text { Infeasibilities (maximum number) } \\
\text { CPU time (s) }\end{array}$ & $\begin{array}{l}\text { NA } \\
\text { NA }\end{array}$ & $\begin{array}{l}\text { NA } \\
\text { NA }\end{array}$ & $\begin{array}{l}896 \\
241\end{array}$ \\
\hline
\end{tabular}



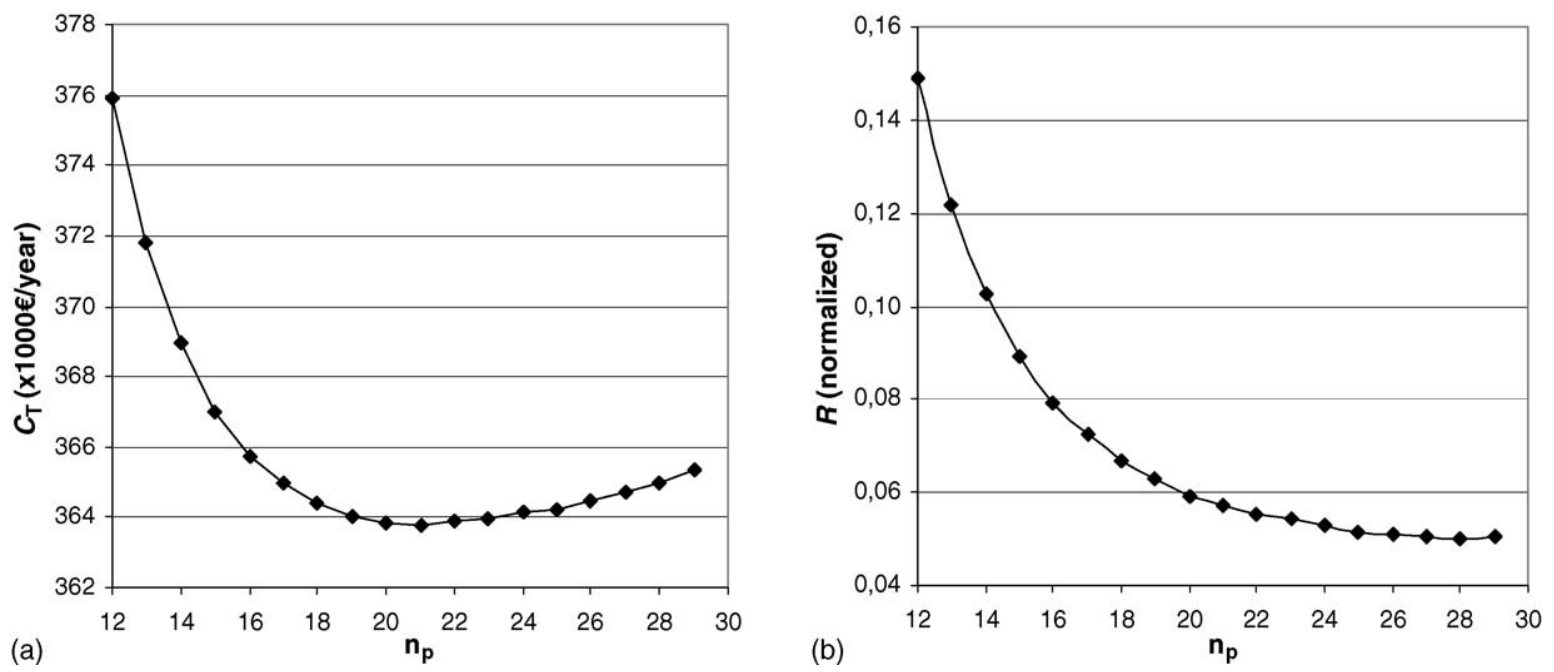

Fig. 9. Variation of the (a) total cost and (b) required reflux ratio with the number of plates (Section 5.4).

\subsection{Example 4}

The objective of the final industrial case study is to synthesize a new distillation unit that could substitute column A in the previous Section 5.2, while simultaneously allowing a $50 \%$ decrease in the actual contamination of the "light" parasite $\mathrm{c}-05$, in stream S3. The selection of the initial number of plates for this column $\left(n_{\mathrm{p}, \max }=30\right)$ originated a very large model, with approximately 17,000 equations/variables.

Economical data from Turton et al. (1998) were also used in this example, in a similar form to the previous case. The three different approaches represented in Fig. 3 were tested with this example. Since the optimal feed location of the new column A was near the top of the column, approaches (b) and (c) of Fig. 3 were the most efficient. Approach (b) was slightly faster than approach (c); the reported convergence data and solution times are therefore relative to it. These results could be anticipated, since an idea of the most favorable region of the column to receive the feed is readily drawn from the shortcut solutions obtained during the pre-processing phase.

This example was tested with several strategies to converge the streams to their optimal locations. Table 10 shows the main convergence results obtained for the three different approaches. In all cases, $123 \mathrm{~s}$ were spent in the pre-processing phase and $187 \mathrm{~s}$ were necessary to obtain the relaxed solution. As can be observed, the combined strategy (variable $\delta$ /variable $\alpha$ ) was able to reduce significantly the number of infeasibilities, when compared with the strategy that used a fixed value of $\delta$; the progression of the optimization procedure is more robust but also slower in this case. The fastest way to converge the problem involves again the fixed $\alpha$ /variable $\delta$ strategy; the number of infeasibilities generated is lower than the fixed $\delta /$ variable $\alpha$ strategy, and the total CPU requirements is lower than when adopting the combined strategy. It was found with this example that the fixed $\alpha$ /variable $\delta$ strategy was also more sensitive to the presence of local optima than the other approaches. For instance, changing the value of $R$ used to initialize the problem in the pre-processing phase was sufficient to influence the value of the final solution obtained in this situation.

The optimal configuration obtained involved a column with 21 equilibrium stages, and the feed located in a single location (Plate 4). The relaxed solution presented the feed stream entering in a single location (Plate 3 ), and the reboil stream splitted $35 \%$ in Plate 19 and the remaining $65 \%$ in Plate 20. These results were confirmed using detailed simulations, shown in Fig. 9.

\section{Conclusions and future work}

This work presented a new continuous formulation for the optimization of complex distillation units, where non-linear programming was used to determine accurately the best locations for the placement of problem streams, together with the optimal number of equilibrium stages and the remaining operational parameters. While similar to the approach of Lang and Biegler (2002), the present formulation can be used to identify additional interesting design configurations for complex distillation units. By retaining a greater similarity between its intermediate solutions and the continuous relaxations of the corresponding discrete formulations, this methodology can combine advantages of previous discrete and continuous approaches. The formulation was applied to several large-scale industrial examples, including the optimization of a set of distillation units. A key to this successful application was the use of a robust pre-processing phase, to initialize, bound and scale the model equations before the start of the optimization phase. The results show that the rigorous optimization of these units can be achieved with NLP solvers, in a reliable manner, resulting in large economical benefits. 
Future developments of this methodology will address the systematic tuning of the adjustable parameters of the formulation during the optimization phase, to enhance the robustness, decrease the total solution time required, and make the approach less sensitive to the presence of local optima.

\section{Acknowledgements}

The authors would like to acknowledge support in different forms from Quimigal, S.A., financial support from Agência da Inovação (AdI), through the consortium research project INOVA and from FCT, through the research project POCTI/EQU/39628/2001.

\section{References}

Barnés, F. J., Hanson, D. N., \& King, C. J. (1972). Calculation of minimum reflux for distillation columns with multiple feeds. Industrial and Engineering Chemistry Process Design and Development, 11, 136.

Barttfeld, M., \& Aguirre, P. A. (2002). Optimal synthesis of multicomponent zeotropic distillation processes 1 , preprocessing phase and rigorous optimization for a single unit. Industrial and Engineering Chemistry Research, 41, 5298.

Barttfeld, M., Aguirre, P. A., \& Grossmann, I. E. (2003). Alternative representations and formulations for the economic optimization of multicomponent distillation columns. Computers and Chemical Engineering, 27, 363.

Bauer, M. H., \& Stilchmair, J. (1998). Design and economic optimization of azeotropic distillation processes using mixed integer nonlinear programming. Computers and Chemical Engineering, 22, 1271.

Biegler, L. T., Grossmann, I. E., \& Westerberg, A. W. (1997). Systematic methods of chemical process design. New Jersey: Prentice Hall.

Brooke, A., Kendrick, D., Meeraus, A., \& Raman, R. (1998). GAMS, a user's guide. Washington: GAMS Development Corporation.

Bruggemann, S., \& Marquardt, W. (2001). Shortcut methods for nonideal multicomponent distillation: Providing initials and bounds for rigorous optimization. Internal report.
EURECHA (2004). The European Committee for Computers in Chemical Engineering Education, Student Contest Problem Competition 2004, <http://www.deb.uminho.pt/escape14/contest.htm>.

Fletcher, R., \& Morton, W. (2000). Initializing distillation column models. Computers and Chemical Engineering, 23, 1811.

Friday, J. R., \& Smith, B. D. (1964). An analysis of the equilibrium stage separations problem-formulation and convergence. AIChE Journal, 698

Grossmann, I. E., Aguirre, P. A., \& Barttfeld, M. (2004). Optimal synthesis of complex distillation columns using rigorous models. In A. Barbosa-Póvoa \& H. Matos (Eds.), European Symposium on Computer-Aided Process Engineering-14 (pp. 53-74). Amsterdam: Elsevier B.V.

Gopal, V., \& Biegler, L. T. (1999). Smoothing methods for complementary problems in process engineering. AIChE Journal, 45, 1535.

Holland, C. (1963). Multicomponent distillation. New Jersey: Prentice Hall.

Lang, Y.-D., \& Biegler, L. T. (2002). A distributed stream method for tray optimization. AIChE Journal, 48, 582.

Nikolaides, I. P., \& Malone, M. F. (1987). Approximate design of multiple-feed/side-stream distillation systems. Industrial and Engineering Chemistry Research, 26, 1839.

Raghunathan, A. U., \& Biegler, L. T. (2003). Mathematical programs with equilibrium constraints (MPECs) in process engineering. Computers and Chemical Engineering, 27, 1381.

Raghunathan, A.U., \& Biegler, L.T. (2004). Interior point methods for mathematical programs with complementarity constraints (MPCCs). SIAM Journal of Optimization, in press.

Reid, R. C., Prausnitz, J. M., \& Poling, B. E. (1987). The properties of gases and liquids (4th ed.). New York: McGraw-Hill.

Seader, J. D., \& Henley, E. S. (1998). Separation process principles. New York: John Wiley \& Sons.

Turton, R., Bailie, R. C., Whiting, W. B., \& Shaeiwitz, J. A. (1998). Analysis, synthesis and design of chemical processes. New Jersey: Prentice Hall.

Viswanathan, J., \& Grossmann, I. E. (1990). A combined penalty function and outer-approximation method for MINLP optimization. Computers and Chemical Engineering, 14, 769.

Viswanathan, J., \& Grossmann, I. E. (1993). An alternate MINLP model for finding the number of trays required for a specified separation objective. Computers and Chemical Engineering, 17, 949.

Yeomans, H., \& Grossmann, I. E. (2000). Disjunctive programming models for the optimal design of distillation columns and separation sequences. Industrial and Engineering Chemistry Research, 35, 1637. 